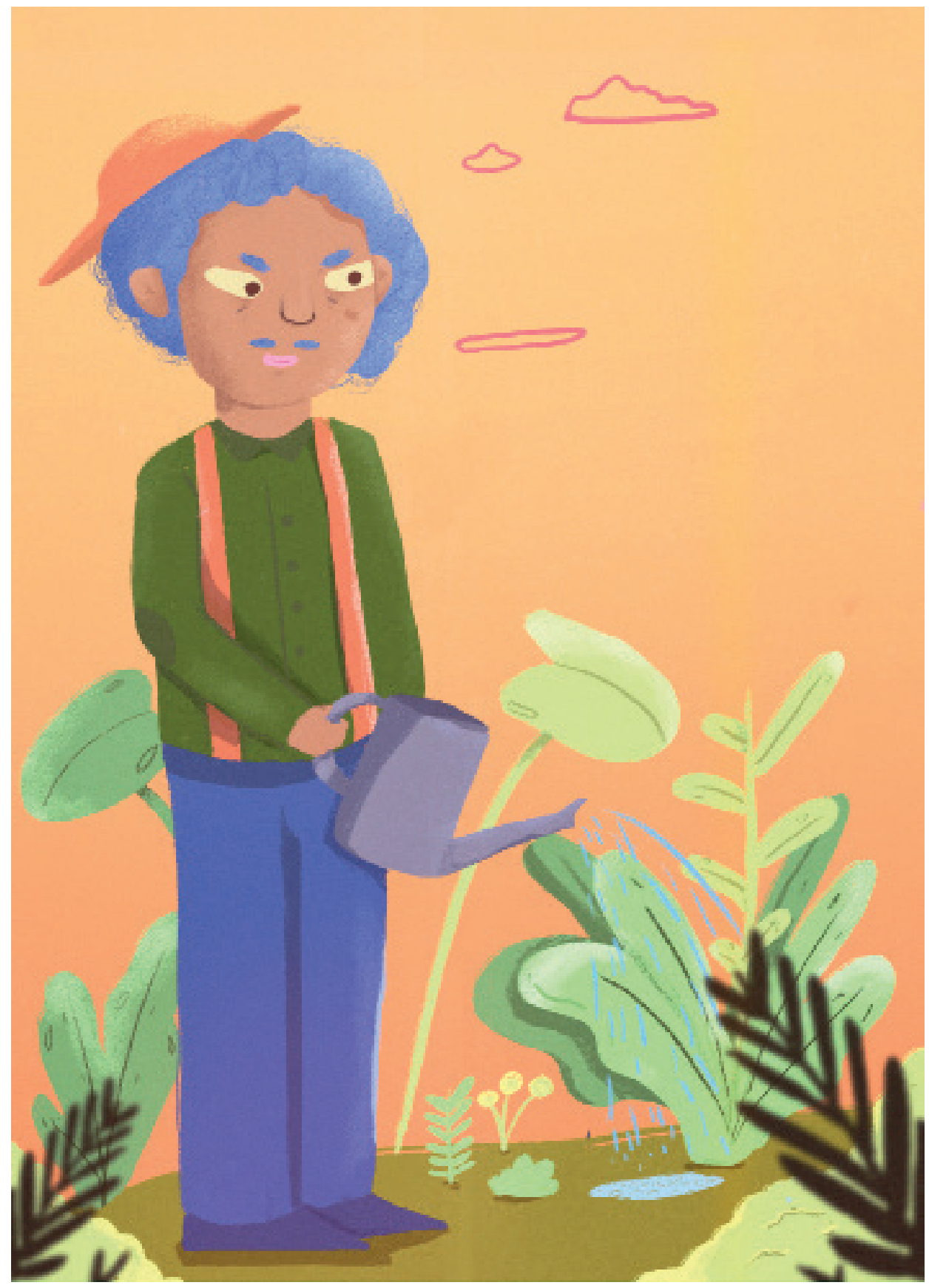




\title{
Retos y dificultades de productores en huertos orgánicos en Celaya, Guanajuato, México'
}

Erandi Delgado González² Perla Shiomara del Carpio ${ }^{4}$ erandirosa@hotmail.com pdelcarpio@ugto.mx

\author{
David Hernández López ${ }^{3}$
}

\section{david.hernandez@itcelaya.edu.mx}

Artículo de investigación recibido el 02/06/2019

y aprobado el 14/07/2019

Cómo citar este artículo:

Delgado González, E., del Carpio Ovando, P. S., \& Hernández López, D. (2019). Retos y dificultades de productores en huertos orgánicos en Celaya, Guanajuato, México. TransPasando Fronteras, (14). doi.org/10.18046/retf.i14.3572

1 Este artículo se realizó en el marco de la investigación "Formas de organización de productores orgánicos para la producción y comercialización en Celaya, Guanajuato", realizada como trabajo de titulación de la Maestría en Estudios Empresariales, Universidad de Guanajuato, Campus Celaya-Salvatierra, División de Ciencias Sociales y Administrativas. Nuestro agradecimiento al Consejo Nacional de Ciencia y Tecnología (CONACYT), por la beca proporcionada en la realización de los estudios de posgrado. 


\section{Resumen}

Esta investigación tuvo como objetivo principal conocer la experiencia de productores orgánicos en Celaya, Guanajuato, México, para analizar sus formas de organización, comercialización y las principales dificultades que afectan al gremio, especialmente, las que se refieren a la producción y comercialización de sus productos. Se realizó bajo un enfoque cualitativo a través de un estudio de caso constituido por un grupo de productores orgánicos perteneciente a un programa gubernamental de desarrollo rural. Se consideró como subunidad de análisis a 18 productores (4 hombres y 14 mujeres) de los 15 huertos que se encuentran distribuidos en 13 localidades del municipio de Celaya. En los resultados se presentan las principales dificultades señaladas por estos productores, tales como: plagas, falta de agua y la poca organización entre integrantes del gremio. Estos se asocian al bajo volumen productivo (que es el principal problema de comercialización), seguido por la falta de tiempo y el desconocimiento de mercado por parte de los productores. Se propone profundizar algunos temas como la importancia del género de los productores, pues se encontró una mayor inclinación de las mujeres por generar ingresos a través de la unidad productiva. Se concluye que la producción orgánica es un espacio de oportunidades para realizar investigación y conocer las luchas y estrategias que los pequeños productores desarrollan ante los impulsos de la globalización.

Palabras clave: Celaya; Comercialización; Organización; Producción orgánica.

2 Estudiante de Maestría en Estudios Empresariales de la Universidad de Guanajuato. Campus Celaya-Salvatierra.

3 Profesor investigador del Instituto Tecnológico de Celaya.

4 Profesora investigadora de la Universidad de Guanajuato. Campus Celaya-Salvatierra 


\title{
Challenges and difficulties of producers in organic gardens in Celaya, Guanajuato, Mexico
}

\begin{abstract}
This research had as main objective to know the experience of organic producers in Celaya, Guanajuato, Mexico, to analyze their forms of organization, commercialization and the main difficulties that affect the union, especially those that refer to the production and commercialization of their products. It was carried out under a qualitative approach through a case study constituted by a group of organic producers belonging to a governmental program of rural development. A subunit of analysis was considered to 18 producers (4 men and 14 women) of the 15 orchards that are distributed in 13 localities of the municipality of Celaya. In the results are presented the main difficulties identified by these producers, such as: pests, lack of water and poor organization among members of the guild. These are associated with low production volume (which is the main marketing problem), followed by lack of time and market ignorance on the part of producers. It is proposed to deepen some issues such as the importance of the gender of the producers, since it was found a greater inclination of women to generate income through the productive unit. It is concluded that organic production is a space of opportunities to carry out research and to know the struggles and strategies that small producers develop in the face of the impulses of globalization.
\end{abstract}

Keywords: Celaya; Commercialization; Organization; Organic production. 


\section{La agricultura orgánica}

La agricultura orgánica ha sido considerada como el maridaje entre el sistema de producción agrícola tradicional y un nuevo sistema de producción. Este término se utilizó por primera vez en 1930 en el libro "Un testamento Agrícola", en el cual se marca el inicio de nuevos métodos de producción sustentado por una serie de observaciones básicas de la agricultura tradicional como su interacción con el medio ambiente, los escasos recursos para producir sin deteriorar, los conocimientos obtenidos por la transmisión generacional y su relación con el medio natural (García y Santiago, 2011).

La agricultura orgánica es entonces un sistema holístico de gestión que cuida la biodiversidad, el medioambiente y la fertilidad del suelo. También cuida las rotaciones sanas de los cultivos con técnicas no contaminantes de bajo empleo de energía y no utiliza pesticidas ni fertilizantes químicos. Este sistema de producción orgánica privilegia el logro de agroecosistemas óptimos y sostenibles, en una dimensión social, ecológica y económica (Codex Alimentarius, 2007).

La Secretaría de Agricultura, Ganadería, Desarrollo Rural, Pesca y Alimentación de México indica que en México la producción de alimentos libres de agroquímicos, antibióticos y transgénicos se ha convertido en una alternativa incipiente para quienes buscan cuidar la salud y el medio ambiente, a esto se le ha denominado: agricultura orgánica (SAGARPA, 2009).

Esta fuente también indica que la agricultura orgánica es un sistema de producción que trata de utilizar al máximo los recursos de la finca, dándole énfasis a la fertilidad del suelo y la actividad biológica y al mismo tiempo permite minimizar el uso de recursos no renovables reduciendo o eliminando el uso de fertilizantes y plaguicidas sintéti- 
cos para proteger el medio ambiente y la salud humana. Derivando de ella distintos tipos de producción como lo son la agricultura urbana y periurbana, hidroponía, cultivos de traspatio, otros. Los productos obtenidos de dichos sistemas de producción obtenidos pueden ser utilizados para el autoconsumo o con fines de comercialización en mercados de agricultores orgánicos (SAGARPA, 2009).

\section{La agricultura orgánica en México y otras experiencias internacionales}

En la experiencia internacional la agricultura orgánica ha sido considerada de gran peso para el futuro pues sus prácticas contribuyen a combatir el cambio climático, ya que aparte de fertilizar la tierra, permite mantener la humedad y la biodiversidad. En términos económicos los cultivos orgánicos superan en rendimiento entre un 80 y $128 \%$ a los cultivos convencionales y en un 20 y $40 \%$ a los ingresos que perciben los campesinos de este mismo tipo de cultivos (Soberanes, 2016). Además, contribuye a la soberanía alimentaria, al producir alimentos sanos, frescos y tradicionales, es decir, sin químicos, herbicidas, pesticidas, hormonas o colorantes (Gee y Haddon, 2016). Según las cifras de Willer y Lernound (2017):

- Las ventas al por menor de alimentos orgánicos y bebidas alcanzaron 81.6 billones de dólares en 2015, incrementando así un 10\% comparado con el año anterior. Ese mismo año se tenían registrados al menos 2.4 millones de productores orgánicos a nivel mundial, es decir, hubo un incremento en más de 160,000 productores en comparación con el año 2014.

- Los países con mayor producción orgánica son India seguido por Etiopia y México, junto con la República Democrática del 
Congo. Perú y Kenia reportaron los incrementos más significativos en los últimos años.

- En la exportación de productos orgánicos sobresalen Holanda, Vietnam y España, mientras que la exportación mexicana de orgánico ocupa el puesto número siete de este ranking.

México sobresale como uno de los productores de orgánicos más importantes del mundo. Sin embargo, de la producción orgánica nacional solamente el 15\% se destina al mercado interno y el restante es comercializado en diferentes países alrededor del mundo. Por ello y debido al pequeño porcentaje de producto ecológico que se comercializa en México, es difícil encontrar comercios en los que se surtan este tipo de alimentos, ya que suelen ser reducidos y específicos. De hecho, si se compara el consumo de productos orgánicos de México frente al del resto de los demás países, es limitado (InfoAgro, 2018).

\section{Productos orgánicos y su comercialización}

La agricultura orgánica puede representar una oportunidad interesante para muchos productores centroamericanos y puede convertirse en una herramienta importante para mejorar su calidad de vida y sus ingresos (FAO, 2003). En un estudio realizado por el Fondo Internacional de Desarrollo Agrícola (FIDA) donde se revisan las experiencias de productores en México y otros países, se encontró que la adopción de la producción orgánica sugiere que hubo efectos positivos sobre la salud de los productores y el ambiente (FIDA, 2003).

En el caso de México la agricultura orgánica se ha caracterizado por la integración de pequeños productores a or- 
ganizaciones privadas o sociales y ha sido mínima la participación del Estado (Schwentesius, Gómez M. y Gómez L., 2016). Schwentesius (2007) realizó un estudio con productores orgánicos de las entidades con mayor representatividad de la producción orgánica (Estado de México, Oaxaca, Morelos, Veracruz, Tabasco y Guerrero) y encontró que uno de los problemas de la integración al mercado de pequeños productores es la falta de independencia de la comercialización de sus productos.

Al solicitar a los productores que definieran un orden de importancia (jerarquía) los problemas técnicos que enfrentan mencionaron, en primer lugar, la falta de técnicos capacitados y, en segundo lugar, la poca oferta de cursos de capacitación. Esta situación desde luego también está relacionada con los problemas institucionales que enfrenta el sector orgánico, que solamente puede resolverse con una fuerte e inmediata participación del Estado y su sistema de educación pública.

Incorporar la agricultura orgánica en el desarrollo rural puede permitir que comunidades rurales y pequeños agricultores puedan acceder a una alimentación saludable a través de su producción agrícola. Puede también generar puestos de trabajo a nivel local (Zamilpa, Schwentesius y Ayala, 2016). El sector orgánico tiene importancia social al estar vinculado con los sectores más pobres del ámbito rural, alrededor del 83\% de los productores orgánicos pertenecen a un grupo indígena y el 99.9\% del total de productores orgánicos son pequeños productores, con tres hectáreas de cultivo en promedio y se encuentran agrupados en organizaciones campesinas. Asimismo, el sector orgánico ofrece alternativas viables de producción y comercialización a los grupos indígenas contribuyendo a la reducción de la pobreza.

La agricultura orgánica en México se ha desarrollado por el esfuerzo de los productores, quienes han contribuido a un desarrollo ru- 
ral incluyente en más de 1000 regiones de producción del país. Por ello, es de vital importancia el apoyo por parte del Estado a través de una visión y estrategia que contemple una política que potencialice los esfuerzos de los productores y considere las ventajas agroecológicas, sociales y culturales para lograr un desarrollo sostenible (Gómez, et. al, 2010).

Para el caso nacional, la problemática del sector orgánico se presenta en cuatro dimensiones. La primera es la dimensión de la producción, esta incluye falta de infraestructura, equipo y transporte, deficiencias agronómicas para el control de plagas y enfermedades, escaso financiamiento para la incorporación a la producción orgánica e, incluso, abandono. La segunda es la dimensión de mercado local, cuya problemática comprende la orientación de la producción, principalmente a aquellos alimentos más rentables en los mercados de exportación; baja organización y capacidad de negociación de los productores para la comercialización, limitado desarrollo de espacios para su comercialización tales como: tianguis, mercados y tiendas especializadas, y restricción de la comercialización solamente a principales ciudades y lugares turísticos(Gómez et al., 2010; Ortigoza, 2010 y Zamilpa, 2014).

La tercera es la dimensión de consumo, incluye escasa información, educación y concientización al consumidor; falta de estrategias de promoción y difusión de bondades; poca disponibilidad de alimentos y baja demanda debido a precios altos y bajo poder adquisitivo del grueso de la población. La cuarta es la dimensión de fomento, abarca la ausencia de políticas públicas orientadas a promover su crecimiento y desarrollo, insuficiente legislación sobre producción orgánica y mayores apoyos políticos y económicos hacia la agricultura convencional (Gómez et al., 2010; Ortigoza, 2010 y Zamilpa, 2014). 
En el caso del estado de Guanajuato (entidad en la que se encuentra la población sobre la que versa este estudio), el sector agroindustrial ha presentado un bajo crecimiento y desarrollo debido a que esta actividad está estrechamente ligada el sector rural. SAGARPA (2010) identificó y examinó las problemáticas, las causas y los efectos que afectan a los agentes económicos con actividad agrícola en el medio rural encontrando las siguientes dificultades: Organización de productores escasa, crédito rural escaso, coordinación e integración de cadenas de valor reducida, mercados ineficientes, gestión de la empresa deficiente, escala de unidades de producción y competitividad reducida.

Dentro de las repercusiones señaladas se encuentran la gestión de la organización deficiente, baja integración de cadenas de valor, mayores niveles de competencia, rendimientos disminuidos, bajo precio de venta, ingresos ineficientes y rentabilidad económica disminuida. Esto podría traducirse en una rentabilidad disminuida en todos los aspectos para el sector rural guanajuatense.

A pesar de ello, los productos orgánicos son cada vez más comunes en la alimentación y forma de vida de la población del estado de Guanajuato. En ranchos y haciendas se encuentran los principales productores de comida libre de químicos, que va directo del productor al comprador. Fue debido a la influencia de presencia extranjera instalada en municipios como San Miguel de Allende que, en la entidad, cada vez son más las personas que adoptan esta forma de alimentación y vida (López, 2016). Municipios como Celaya han tenido una posición clave para la industria del sector primario y terciario en el estado de Guanajuato, pues pertenece a un corredor industrial con corporativos nacionales y multinacionales, y cuenta con acceso a las rutas de Comercio Internacional con Asia, Estados Unidos, Centro y Sur de América. 
En Celaya se realiza escasa actividad agrícola y, generalmente, los espacios de producción se encuentran en la periferia de la cabecera municipal, como es el caso de la producción sobre la que versa esta investigación. El municipio presenta un agotamiento y desabasto de agua, que no deja de afectar a sus habitantes, particularmente a los pequeños productores rurales que usan el agua para la irrigación de las pequeñas parcelas (López y Hernández, 2016; Secretaria de Desarrollo Social, 2010).

Por ello, conocer el contexto, los retos, las dificultades y la situación de comercialización agrícola de productores orgánicos de Celaya, permite la creación de propuestas de acción encaminadas a la mejora de un sector que impulsa la economía local a través de su oficio (a veces poco valorado y reconocido). Esto permite, además, conocer las múltiples luchas en conjunto que enfrentan los productores orgánicos en el ámbito rural.

\section{Metodología}

\section{Enfoque y técnicas}

Este estudio acude a la metodología cualitativa y constituye un estudio de caso que centra la atención en un grupo de productores orgánicos perteneciente a un programa gubernamental de apoyo rural. De dicho programa se desprende un proyecto de huertos orgánicos que consta de la instauración de uno o más huertos en diferentes localidades rurales del municipio de Celaya (perteneciente al Estado de Guanajuato, entidad localizada en el centro de la República Mexicana), localidades con algún tipo de rezago social.

A los productores se les otorga sustento técnico, infraestructura (ecotecnologías y semillas) y capacitación, así como sustento para 
comercializar sus productos (hortalizas, frutas, verduras). En el caso que aquí nos ocupa, en el grupo de productores sobresale la presencia de mujeres, madres de familia y personas de la tercera edad.

Las técnicas a las que se ha recurrido son: entrevista semiestructurada, diario de campo y revisión documental. El trabajo de campo se realizó los viernes y ocasionalmente los jueves durante el periodo del 9 de febrero al 30 de marzo de 2018, en las comunidades de San Isidro de Trojes, Nuevo Porvenir, San José de Yustis, Los Mancera, Santa Anita, Juan Martín, Arreguín de Abajo, El Cuije, San Miguel Octopan, Jauregui, La Aurora y San Elías. En la mayoría de estas comunidades existe 1 huerto, sin embargo, en San Miguel Octopan, San Elías y La Aurora hay 2 huertos. A continuación, se presenta un mapa de la cuidad de Celaya con la ubicación geográfica de las localidades en las que se ubican los productores participantes en este estudio.

Imagen 1 Localización geográfica de huertos en el municipio de Celaya

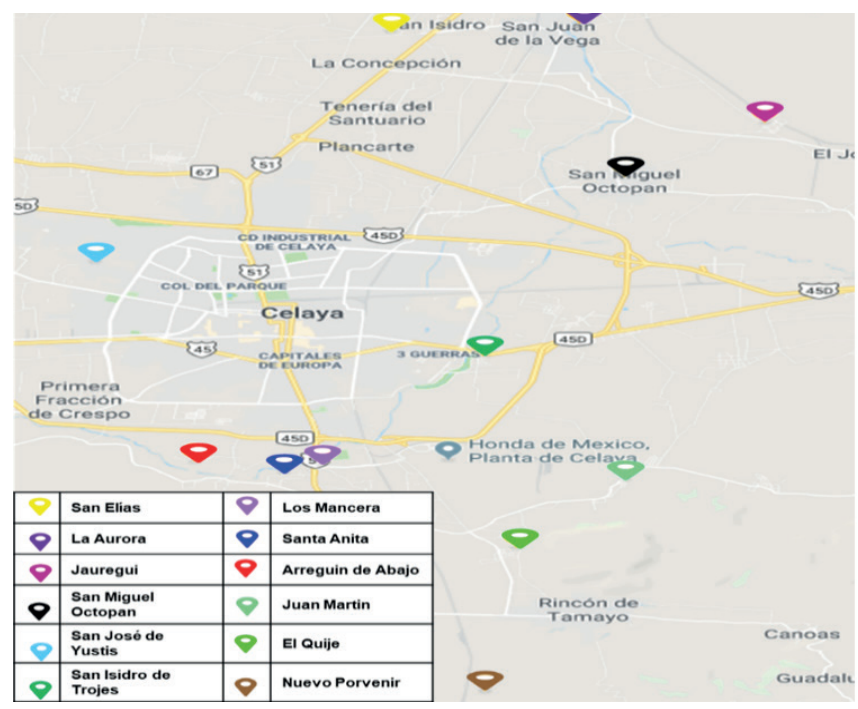




\section{Población}

El promedio de edad de la población participante es de 35 años, la mayoría con estado civil de casado. Se contó con la participación de 18 productores: 14 mujeres y 4 hombres. Los participantes del sexo masculino presentan homogeneidad en cuanto a su nivel de estudio, pues cursaron la educación primaria y su estado civil es casado. Mientras que las mujeres muestran mayor heterogeneidad, ya que el nivel de estudios promedio se encuentra dividido entre la primaria y secundaria (5 en cada uno) y 4 en preparatoria. El estado civil de la mayoría es de casada (10 mujeres), participaron también 2 solteras, 1 divorciada y 1 mujer en situación de unión libre.

\section{Resultados y discusión}

Para el análisis de los resultados se consideraron categorías de análisis construidas a partir del contenido de las entrevistas realizadas. Las temáticas abordadas, desde las cuales se crearon categorías de análisis, fueron: experiencias, problemáticas y disposición para participar en actividades comerciales. Respecto a la comercialización los entrevistados señalaron que en 7 de los 15 huertos se han vendido los productos que han cultivado mientras que 8 no lo han hecho. Entre los productos que han vendido se encuentran, principalmente, hortalizas: lechuga, cilantro, chiles y acelgas, además de calabaza y plántula de ciertas especies como jitomates, apio, entre otras (véase figura 2). Los siguientes testimonios señalan dicha producción:

"Sí, hemos vendido parte de nuestras cosechas como cilantro, cebolla, lechuga" (Hombre, 56 años, casado, Los Mancera).

"Sí, (vendo) cilantro, calabazas" (Mujer, 48 años, casada, El cuije). 
La decisión de los productores de comercializar este tipo de productos ha sido acertada pues como lo señala la Secretaría de Desarrollo Agroalimentario y Rural Guanajuato busca posicionarse en el primer lugar de producción orgánica de hortalizas (SDAyR, 2018). Solamente en 2017 se exportaron alrededor de 24.424 hectáreas de producción orgánica, por lo que en un futuro los productores de los huertos orgánicos de este estudio podrían estar sumándose a estas cifras ya que, por el momento, al no estar certificados, no pueden hacerlo. Sin embargo, estos productores pueden comercializar sus productos de manera local.

Figura 2 Especies de productos vendidos por los productores de Celaya

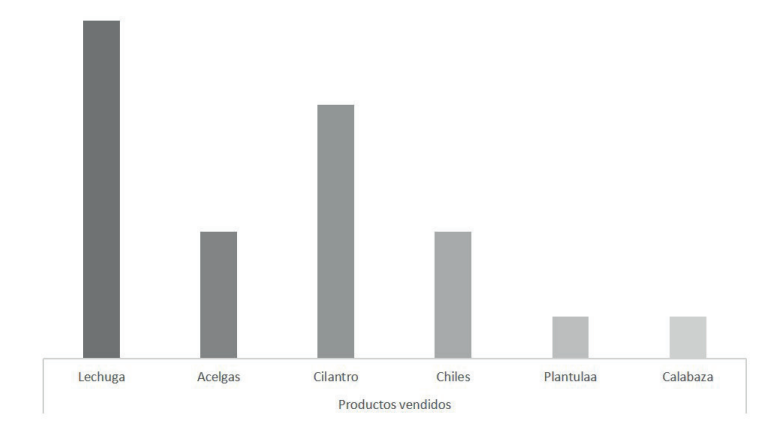

Fuente: Elaboración propia a partir del trabajo de campo realizado.

Debido a que los productores señalan haber tenido experiencia vendiendo sus productos, fue necesario saber por qué vías lo habían logrado. Se tuvieron respuestas variadas, entre las que se encuentran: Ventas casa por casa con sus vecinos (cambaceo) o por las calles de su comunidad; la venta a pequeños comerciantes como taqueros, abarroteros, marisquerías, etc. Mientras que en algunos casos han sido partícipes de exposiciones donde se les ha dado la oportunidad de vender sus productos. Otros, por su parte, han comercializado en su propio negocio. 
Figura 3 Tipos de ventas

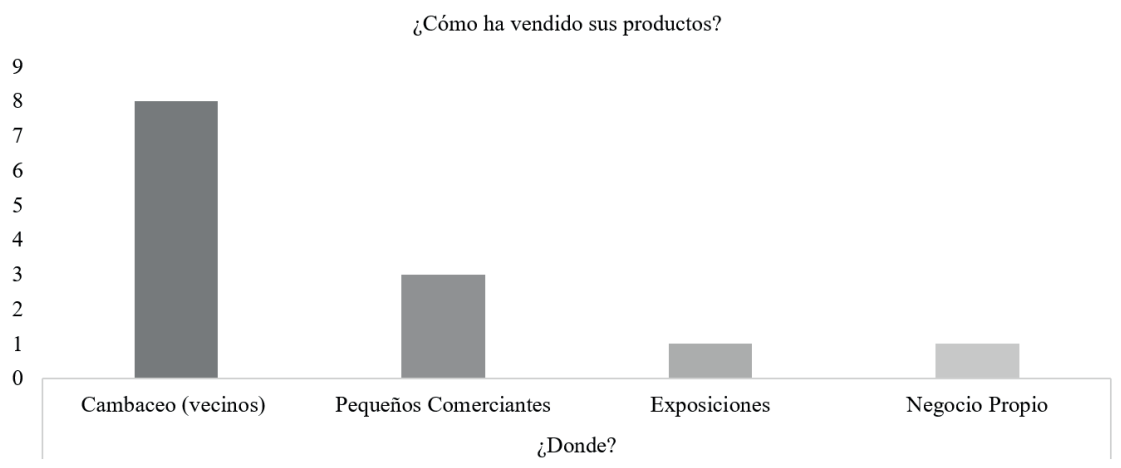

Fuente: Elaboración propia a partir del trabajo de campo realizado.

Los siguientes relatos describen las circunstancias en la que los productores han comercializado sus productos:

"Con las vecinas (vendo). Ellas me encargan o a veces yo les ofrezco de lo que tengo" (Mujer, 64 años, casada, La Aurora).

"Aquí en la comunidad (vendo) y en una marisquería que fue una de las ventas más grandes" (Hombre, 70 años, casado, Santa Anita).

En algunos casos, la calidad de los productos que han ofrecido ha hecho que los vecinos empezaran a buscar a los productores para comprar lo que ellos ofertan. Lo anterior se constata en el siguiente fragmento de entrevista:

"Pues antes lo que producíamos de más lo ofrecía aquí en la tienda. Les decía que era de nuestro huerto y que podían estar más chiquitos que los que usualmente vendemos y si, los empezaron a comprar. Pero luego mi sorpresa fue que, aunque hubiera, me preguntaban que si no tenia de mi huertito que porque les habían gustado mucho" (Mujer, 44 años, soltera, San José de Yustis). 
Teniendo en cuenta lo anterior, es posible que la forma de comercialización de los productos tenga que ampliarse o modificarse. Esto debido a que un producto sea comercialmente reconocido como orgánico es necesario que incluya el sello de certificación que garantice que todos los insumos involucrados en su producción son orgánicos y libres de cualquier sustancia química que los contamine (PROFECO, 2018). Estas certificaciones representan una oportunidad de apertura en el mercado tanto nacional como internacional.

Los inconvenientes percibidos por los entrevistados al momento de intentar vender sus productos (véase figura 4), indican que varios de ellos se encuentran ligados directamente a las problemáticas de producción como los bajos volúmenes productivos.

"Sentimos que aún no producimos tanto como pues pa' (para) sacar una buena ganancia" (Mujer, 42 años, divorciada, Jauregui).

Analizando esta respuesta se podría observar que no difieren mucho de la situación actual que viven otros productores en el país, ya que -como señala SAGARPA (2014)-, las principales dificultades del entorno agrícola se encuentran relacionadas con la falta de tecnología, la asistencia técnica limitada, la baja disponibilidad de insumos orgánicos, la dificultad en garantizar el cumplimiento de métodos orgánicos, la certificación obligatoria y costosa, los mercados limitados con altas exigencias, los procesos de reconversión largos y costosos, la dificultad para renunciar a los insumos químicos y la reducción del uso de maquinaria. Otras dificultades señaladas por las personas entrevistadas son robos, saqueos y daños ocasionados por actores externos a la producción y la "falta de tiempo" de los huertistas. Este último surge debido a que tienen otras actividades que cumplir dentro y fuera del hogar, lo que dificulta un balance entre las actividades que realizan para obtener ingresos. 
"Tiempo es lo que a veces hace falta, más que nada por la familia" (Mujer, 31 años, casad a, San Elías).

"Tiempo, porque ahorita nosotros vamos iniciando y pues ahorita hay que ver cómo repartir las actividades en el día" (Mujer, 50 años, casada, Juan Martin).

Figura 4 Problemáticas que afectan a la comercialización

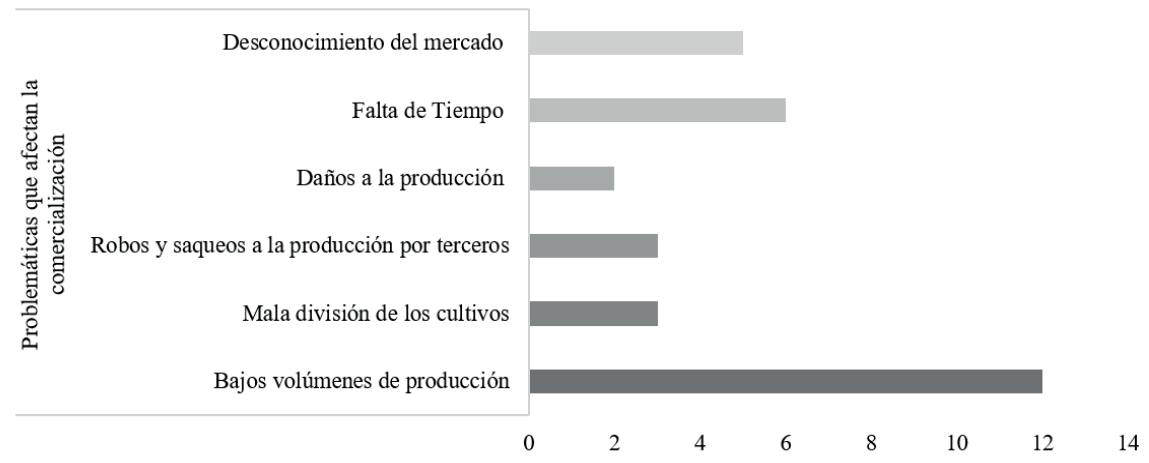

Por otro lado, los productores indicaron que las 3 características más importantes para mejorar o llevar a cabo la comercialización, son: asistencia técnica, acceso al agua e incrementar los volúmenes de producción. Estos aspectos se relacionan completamente con el área productiva. 
Figura 5 Posibles mejoras para la comercialización

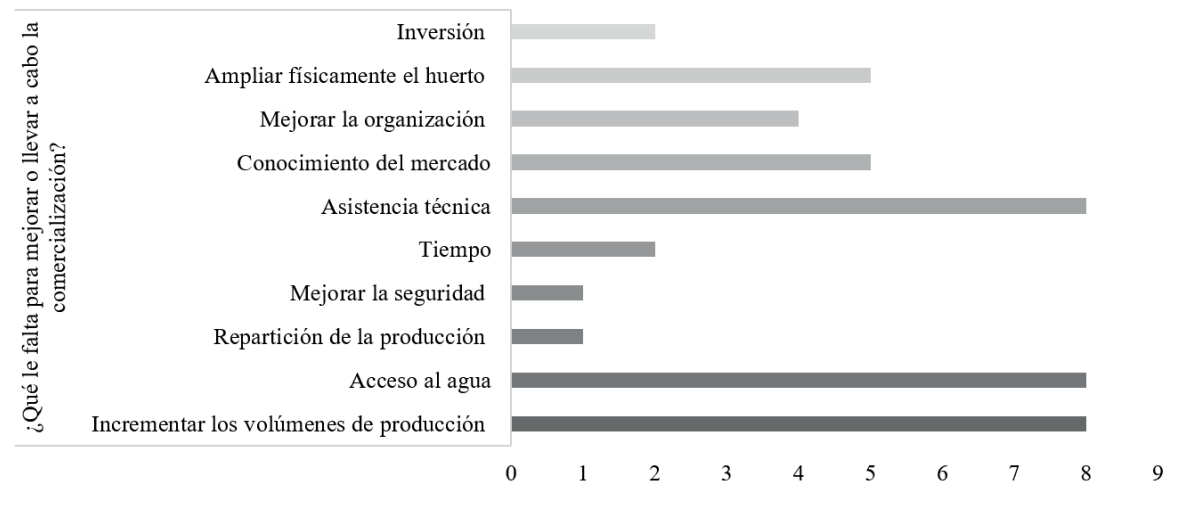

Fuente: Elaboración propia a partir del trabajo de campo realizado.

Para algunos de los productores es preocupante el poco o nulo "conocimiento del mercado" que tienen, lo cual provoca que sientan incertidumbre de saber a quién y/o dónde podrían ofertar sus productos para poder recibir un pago justo por el trabajo realizado.

"Pues no sé quién lo consumiría, a quién le interesaría comprarlo y también pues que lo paguen bien y no se malbaraten" (Mujer, 50 años, casada, Juan Martin).

"No he sabido aplicar un precio justo para mis productos y pues es necesario". (Hombre, 70 años, casado, Santa Anita).

Zamilpa (2014) respecto al panorama nacional y haciendo alusión a las problemáticas del sector agrícola indica que: 
La poca organización de los productores y producción a pequeña escala es una causante de muy alta importancia (41.2\%) para la problemática del mercado local orgánico en México. Este resultado es consistente con el hecho de que a los pequeños productores les es difícil conformar una oferta que abastezca el mercado interno en función de sus características (p. 20).

Por ello, es trascendente retomar las estructuras organizativas derivadas de la economía social (como la cooperativa) que los proveerá de autogestión, apoyo mutuo, autonomía, equidad dentro de sus labores y el comercio justo para regular los precios de mercado entre los grupos con distinta condición económica. Esto con el fin de lograr una expansión adecuada en el mercado y mantener un nivel competitivo en este, sin dejar de lado la rentabilidad.

Y es por estas necesidades que instituciones como la Red Mexicana de Tianguis y Mercados Orgánicos AC (REDAC), promueven la compraventa, la sostenibilidad y la justicia económica y social. Teniendo como objetivos:

- Fomentar la producción y el consumo de productos orgánicos, el comercio justo y solidario entre los participantes de la red.

- Promover, apoyar y asesorar la reconversión de productos convencionales y naturales a orgánicos.

- Organizar y fomentar actividades de formación a través de cursos, talleres, conferencias y otros medios.

- Promover y asesorar en materia de "Certificación Participativa" (CP), con la finalidad de garantizar el origen y la calidad orgánica de los productos que se ofrecen en cada tianguis y/o mercado (Schwentesius, 2015). 
Otras dificultades mencionadas por los productores y que no han sido encontradas en otros estudios son la "falta de tiempo" y "la mala distribución de los cultivos". Esto se debe a que no se tenía previsto que el cuidado del huerto podría considerarse un problema que llegara a afectar en el día a día de los responsables o que el reparto de los cultivos fuera causante de conflictos.

"Pues apenas alcanza para repartirlo entre nosotros y luego hay algunas gentes que se quedan más" (Hombre, 56 años, casado, San Miguel Octopan).

Participar de una actividad comercial como algún tianguis o exposiciones que les generen ingresos resulta, para la población de este estudio, un tema que divide las opiniones entre los huertistas. 8 de los 15 huertos se presentan abiertos a la posibilidad y lo reafirman a través de la exposición de las razones que los llevarían a hacerlo, como el reconocimiento (motivación), la generación de vínculos y la necesidad de obtener ingresos. Por ejemplo:

"Sí (lo haría), para vender y que me conozcan, compartir experiencias y ganar dinero" (Mujer, 48 años, casada, El cuije).

Otros aún parecen ser más cautelosos (4 de ellos) al señalar que esto podría llegar a ser "en otro momento", ya sea por razones familiares o por la percepción de falta de tiempo, a la vez que reconocen que estas circunstancias son temporales y podrían modificarse en cierto plazo de tiempo:

"En otro momento, pues porque ahorita mis niños están muy pequeños y pues salir a vender lleva tiempo, pero ya estando ellos en la primaria que están más horas pues si me animaré" (Mujer, 26 años, casada, San Elías). 
Y, por último, encontramos a tres huertistas que tienen nula disposición para participar en alguna de actividad comercial. Dentro de sus razones señalan falta de motivación y desconfianza en sus propias capacidades (para poder comercializar sus productos), además de que consideran que la edad ya no se los permitiría.

"No, bueno, yo no podría por mi edad y porque no sabría cómo venderlos, pero pues si alguien más del grupo es bueno pues adelante" (Hombre, 72 años, casado, Los Mancera).

Si bien es válido que los productores se sientan recelosos, cautelosos o desconfiados respecto a la comercialización, una posible motivación para tomar en cuenta la comercialización de sus productos yace en que ha ganado cierta popularidad en el país, tanto en supermercados como en restaurantes. Los alimentos orgánicos se presentan con mayor prestigio gracias al beneficio para la salud y medio ambiente. El mercado interesado en este tipo de productos crece 10\% anual en México, pues existe una tendencia creciente en la demanda por parte de las familias mexicanas. Además, este tipo de productos suelen tener una mayor utilidad hablando en términos económicos que los convencionales (Esnaurrizar, 2017), lo que ayudaría al productor orgánico a garantizar buenas ganancias y volúmenes estables de compra en sus productos, disminuyendo la incertidumbre. Falta, sin duda, compartir esta información con los productores orgánicos.

Como consecuencia del análisis de la situación de los productores en los huertos orgánicos de la cuidad de Celaya en tema de comercialización, sobresale que muchas de las problemáticas que tienen los huertistas para comercializar son derivados de la producción, además de la necesidad de información y capacitación respecto a regulación de precios, criterios de calidad y canales de comercialización. Se enfrentan a la situación mundial que señala la FAO (2003a) respecto a las cantidades limitadas de productos orgánicos y las normas de cali- 
dad que se demandan a los productores, al igual que las normas que rigen la producción. Lo anterior podría limitar a los países en vías de desarrollo en su capacidad para satisfacer la demanda de alimentos orgánicos. En ese contexto cobra mayor importancia que los huertistas de este estudio hayan empezado adquirir experiencia comercializando sus productos por cuenta propia, a la vez que reconocen que requieren mayor capacitación en la producción y la comercialización de sus productos, pues saben que su trabajo también es atravesado por cuestiones estructurales que sobrepasa su voluntad y su capacidad de producción. Deberán enfocarse en dos elementos fundamentales: los procesos de apropiación de conocimiento y en gestar nuevos conocimientos y procesos (Amador, 2003).

\section{Conclusiones}

El grupo de productores orgánicos sobre los que versa este estudio presenta particularidades que lo diferencian de otros productores. Por ejemplo, el hecho de que nació de un proyecto gubernamental que apoyó a la constitución del grupo y al crecimiento de sus unidades productivas; esto a través de semillas, ecotecnologías (tecnologías para satisfacer las necesidades humanas, minimizando el impacto ambiental) y algunas capacitaciones técnicas. Conjuntamente este grupo tiene la ventaja de encontrarse en una ciudad con una ubicación estratégica que los conecta con rutas de comercio nacional e internacional importantes, que en un futuro podrían ser aprovechadas para comercializar sus productos. Por ello, se considera vital la estrategia de consolidación de una figura organizativa que promueva el sentido de filiación de los productores y refuerce la importancia de su participación en las acciones comerciales, pues contar con motivación constante y un respaldo organizacional disminuye el abandono de la unidad productiva e 
incentiva su crecimiento y mejora sus rendimientos. Es preciso también impulsar economías solidarias como el comercio justo en la cuidad de Celaya debido a que conjunta la movilización social y la incidencia política que, al mismo tiempo, pudiese promover la reducción de la pobreza, pues los pequeños productores pudieran ser autosuficientes gracias a esta iniciativa.

Esto debidamente aplicado estaría cerrando el camino para amenazas como la competencia, la baja rentabilidad agrícola, además de abordar los índices de pobreza rural, mejoramiento de las políticas públicas y desarrollo rural urbano. Se considera importante también señalar que el estado de Guanajuato sobresale en el panorama nacional por su producción agrícola, por ello es de vital importancia realizar la apuesta a la revitalización del campo mexicano y, en este caso, a la producción orgánica de la región de estudio, tomando como estrategias la corrección de incentivos para que dejen de beneficiar únicamente a las empresas industriales, específicamente a las trasnacionales, y ampliar la orientación educativa hacia el campo, esto para promover nuevas visiones empresariales e incentivar la creación y fortalecimiento de las cadenas productivas. Sin duda, es importante propiciar y promover la capacitación en producción y comercialización de estos pequeños productores de hortalizas; seguir realizando investigación multidisciplinaria respecto a estas iniciativas que, aunque hechas en una dimensión micro reflejan también luchas y tensiones en una dimensión macro que obedecen a condiciones estructurales de nuestro país. Para intentar cerrar, solamente señalar que consideramos que la producción orgánica es un espacio de infinitas oportunidades para conocer las luchas y estrategias que los pequeños productores desarrollan ante los impulsos de la globalización. Estudiar sus procesos de cambios y permanencias siempre será un acierto. 


\section{Referencias}

Amador, M., (2003). Comercialización de productos orgánicos, gestión de procesos agroecológicos: Una experiencia de aplicación. En: Agricultura Orgánica: una herramienta para el desarrollo rural sostenible y la reducción de la pobreza. Recuperado de: https://bit.ly/2Gf3g67

Codex Alimentarius (2007). Alimentos Producidos Orgánicamente. Recuperado de: https://bit.ly/38zm4Jk

Cussianovich, P. y Altamirano, M. (2005). Estrategia nacional para el fomento de la producción orgánica en Nicaragua. En Cooperación para el Desarrollo. Recuperado de: https://bit.ly/3aDjHXO

Esnaurrizar, F. (1 de septiembre de 2017). Alimentos orgánicos, tendencia e inversión. El economista. Recuperado de: https:// https://bit.ly/3810tyP

Fondo Internacional de Desarrollo Agrícola [FIDA] (2003). La Adopción de la agricultura orgánica por parte de los pequeños productores. Recuperado de https://bit.ly/2TNY6pR

García D. y Santiago M. (2011). Alimentos ecológicos, Alimentación Sana. Recuperado de: http://www.grefa.org/grefa/alimentos_ecologicos.pdf

Gee K. y Haddon H. (2016). Los grandes salen a la caza de los alimentos orgánicos, en The Wall Street Journal Americas, 11 de julio.

Gómez M, A., Schwentesius, R., Ortigoza, J., L, G., y Tzun, M. (2010). Agricultura, apicultura y ganadería orgánicas de México, 2009: estado actual, retos, tendencias. Recuperado de: https://bit.ly/38vkM21

InfoAgro (2018). El mercado de los productos orgánicos. Recuperado de: https://bit.ly/38zmE9Y 
López, C. (2016). Crece mercado orgánico. Recuperado de: https://bit.ly/2GgpOyC

López, P.; Hernández, J. (2016). Caso de estudio Celaya. En Programa Cohesión Territorial para el Desarrollo. Rimisp, Santiago, Chile. Recuperado de: https://bit.ly/2unmDr2

Mancera, F. (6 de noviembre de 2018). Crean productores tianguis orgánico. Periódico A.M. Recuperado de: https://bit.ly/2RDKACq

Organización de las Naciones Unidas para la Agricultura y la Alimentación [FAO] (2003). ¿Es la certificación algo para mí? Recuperado de: https://bit.ly/3aAlhZo

Organización de las Naciones Unidas para la Agricultura y la Alimentación [FAO] (2003a). Perspectivas de la agricultura orgánica. En Agricultura Orgánica, Ambiente y Seguridad Alimentaria. Recuperado de: http://www.fao.org/3/y4137s/y4137sOf.htm

Procuraduría Federal del Consumidor (2018). Alimentos orgánicos. Recuperado de: https://bit.ly/2TNeruL

Sauza, J. L. (2016). Valoración del proceso del plan piloto, Manuscrito presentado para su publicación. Secretaría de Desarrollo Agroalimentario y Rural Celaya, Guanajuato, México.

Secretaría de Agricultura, Ganadería, Desarrollo Rural, Pesca y Alimentación [SAGARPA] (2009). Las organizaciones económicas del sector rural: Principios y bases jurídicas. Recuperado de: https://bit.ly/2RHbZmT

Secretaría de Agricultura, Ganadería, Desarrollo Rural, Pesca y Alimentación [SAGARPA] (2010). Diagnóstico sectorial en el Estado de Guanajuato. Informe Preliminar (Borrador). Recuperado de: https://bit.ly/2RiSFNR 
Secretaría de Agricultura, Ganadería, Desarrollo Rural, Pesca y Alimentación [SAGARPA] (2014). México Calidad Suprema. Recuperado de http://www.mexicocalidadsuprema.org/archivos/senasica.pdf

Secretaría de Desarrollo Agroalimentario y Rural [SDAyR] (2018). La Agricultura Orgánica Es Gran Área De Oportunidad. Recuperado de: https://bit.ly/2TR2S5Q

Secretaría de Desarrollo Social [SEDESOL] (2010). Catálogo de localidades. Recuperado de: https://bit.ly/30NvzlS

Schwentesius, R. (2007). Problemática del Sector Orgánico Mexicano. Recuperado de: https://bit.ly/37kkuen

Schwentesius, R., Gómez Cruz, M.,y Gómez Tovar, L. (2016).Agricultura Orgánica en México 2016. Recuperado de: https://bit.ly/2TP4UTX

Soberanes, M. (2016). Producción orgánica certificada. Avances en el sistema de información, Ponencia presentada en el Encuentro de Productores, Procesadores, Comercializadores y Consumidores de Productos Orgánicos, México, junio (p.54).

Willer, H., Lernound, J (2017). The World of organic Agriculture Statics and Emering Trends- Organics International. Recuperado de: https:// shop.fibl.org/CHde/mwdownloads/download/link/id/785/?ref=1

Zamilpa, J. (2014). Fortalecimiento del sector orgánico de México: Aprendiendo de la experiencia de la unión europea. (Tesis doctoral) Recuperado de: http://www.eumed.net/tesis-doctorales/2015/jzp/

Zamilpa, J., Schwentesius Rindermann, R. y Ayala Ortiz, D. A. (2016). Estado de la cuestión sobre las críticas a la agricultura orgánica. Acta Universitaria, 26 (2), pp. 20-29. doi: 10.15174/au.2016.854. 